\title{
Erratum to: The laminar organization of the motor cortex in monodactylous mammals: a comparative assessment based on horse, chimpanzee, and macaque
}

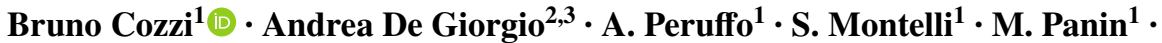 \\ C. Bombardi ${ }^{4}$ A. Grandis ${ }^{4}$ A. Pirone ${ }^{5}$ P. Zambenedetti ${ }^{6} \cdot$ L. Corain $^{7}$. \\ Alberto Granato ${ }^{2}$
}

Published online: 18 April 2017

(C) Springer-Verlag Berlin Heidelberg 2017

\section{Erratum to: Brain Struct Funct \\ DOI 10.1007/s00429-017-1369-3}

The second co-author name has been published incorrectly in the original publication of the article. The correct coauthor name should be "Andrea De Giorgio".

The online version of the original article can be found under doi:10.1007/s00429-017-1369-3.

Bruno Cozzi

bruno.cozzi@unipd.it

$\triangle$ Alberto Granato

alberto.granato@unicatt.it

1 Department of Comparative Biomedicine and Food

Science, University of Padova, Viale dell'Università 16, 35020 Legnaro, PD, Italy

2 Department of Psychology, Catholic University of the Sacred Heart, Largo A. Gemelli 1, 20123 Milan, MI, Italy

3 Faculty of Psychology, eCampus University, Novedrate, CO, Italy

4 Department of Veterinary Medical Sciences, University of Bologna, Ozzano dell'Emilia, BO, Italy

5 Department of Veterinary Sciences, University of Pisa, Pisa, PI, Italy

6 Pathology Division, General Hospital of Dolo-Venezia, Dolo, VE, Italy

7 Department of Management and Engineering, University of Padova, Vicenza, VI, Italy 